\title{
Listeria monocytogenes Infects the Zebrafish Central Nervous System; A Useful Model to Analyze the Neuroimmune Response
}

\author{
Christopher P. Corbo ${ }^{1}$, Corey E. Gaylets ${ }^{1}$, Alex J. Molesan ${ }^{1}$ and William A. Rivera ${ }^{1}$ \\ ${ }^{1}$ Microbiology Program, Department of Biological Sciences, Wagner College, Staten Island, NY 10301
}

Listeria monocytogenes is a facultative, intracellular, gram-positive bacterium that causes listeriosis in immunocompromised patients, causing death in 20\%-30\% of infected individuals [1,2]. In severe cases, this pathogen has been shown to enter the central nervous system (CNS). Usually, Listeria monocytogenes is ingested from contaminated food, particularly seafood, soft cheeses, and meat, being that this organism grows well at a wide range of temperatures [3]. From a cellular perspective, this pathogen has developed an array of molecular mechanisms by which it is able to invade a host cell, thrive and replicate before migrating to subsequent cells all while evading the immune system of the host organism [4]. In short, the infection begins when the bacterium is phagocytized by macrophages, where rather than being destroyed, using listeriolysin $\mathrm{O}$, it escapes the $\mathrm{m}$ vesicle and survives in the host macrophage. From here, the bacterium proliferates and spreads to other cells in the organism.

Our work set out to investigate if zebrafish could be used as a model organism to study pathogenesis of Listeria monocytogenes specifically in the CNS. Zebrafish have become more widely accepted as models in which to study bacterial pathogenesis [5]. There have been few studies which have investigated Listeria pathogenesis both in larval zebrafish [6] and in adult zebrafish [7]. Both studies have shown that zebrafish are, in general, more resistant to L. monocytogenes infection than other mammalian models used. However, neither study investigated the ability of the organism to infect the zebrafish CNS. The goal was to establish and characterize a model where the CNS of adult zebrafish was infected with Listeria monocytogenes. To accomplish this, L. monocytogenes was injected into the vitreous humor of the zebrafish eye. The fish were sacrificed on a time course and their brain was removed and prepared for either microscopy, bacterial enumeration or molecular detection. We hypothesized that the Listeria monocytogenes could enter the cells of the retina and move into the CNS by way of the optic nerve.

Approximately $50 \%$ of the injected fish survived 24 hours. This increased to around $75 \%$ in later experiments, suggesting that there is a learning curve for the investigator performing the experiment. At 24, 48, 96 hours and 7 days after the injection, fish were sacrificed under anesthesia and their brains were plated on Oxford agar. Initially, fish that died prior to the first 24 hours were not positive for the presence of $L$. monocytogenes. Brains of fish sacrificed later than 24 hours were all positive for $L$. monocytogenes.

At the light microscopic level, cells of the periventricular grey zone (PVGZ) were seen to be abnormally shaped and unhealthy in appearance. Transmission electron microscopy revealed the presence of Listeria in the optic tectum of the zebrafish brain. Specifically residing in and among the cells of the PVGZ. This region of the brain is the direct input of the optic nerve and the major visual processing cortex of the zebrafish [8]. Listeria cells were easily detected between the cell bodies of the PVGZ. The cells of this region were also, in some cases damaged, appearing as if a hole was punched into the side of the cell. Additionally, the characteristic "escape route" of Listeria was detected in the cells of this region (Figure1). 
Using western blotting (Figure 2), the brain of zebrafish was positive for the presence of Listeria monocytogenes cells after 48 hours of infection and was positive for all time points up to the final point at 7 days post injection. Additionally, blots from 48 hours and 7 days post injection were also positive for ActA, the actin recruitment virulence factor. Cells grown in culture were not positive for ActA suggesting that in the zebrafish brain tissue, L. monocytogenes is virulent.

This work has demonstrated that via injection into the eye, Listeria monocytogenes is able to move into the CNS and initiate an infection. It was also shown that not only is the Listeria present in and around the cells of the PVGZ, but they are also causing damage to the cells of this brain region. Future work will use this model to study the neuro-immune response of the zebrafish and the interaction of $L$. monocytogenes with the host cells.

\section{References}

[1] AS Angelidis and K Koutsoumanis, J. Food Prot. 69 (2006), p. 938-42

[2] PN Skandamis et al, Food Microbiol. 25 (2008) p. 294-303

[3] DM Kagkli et al, Applied and Enviornmental Microbiology 75 (2009), p. 3621-6

[4] DA Portnoy et al, The Journal of Cell Biology 158 (2002), p. 409-14

[5] AM van der Sar et al, Trends in Microbiology 12 (2004), p. 451-6

[6] JP Levraud et al, Infection and Immunity 77 (2009), p. 3651-3660

[7] A Menudier, FP Rougier, and C Bosgiraud, Path Biol. 44 (1996), p. 783-789

[8] CP Corbo et al, Microscopy Research and Technique 75 (2011), p. 325-333
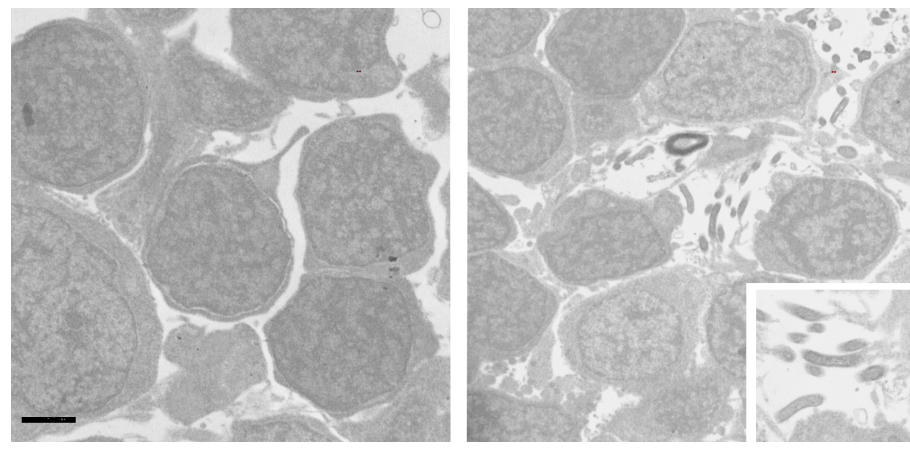

Figure 1 - Periventricular grey zone cells from zebrafish infected with L. monocytogenes. The left panel shows Listeria pushing out of a host cell while the right side shows Listeria cells in the surrounding environment.

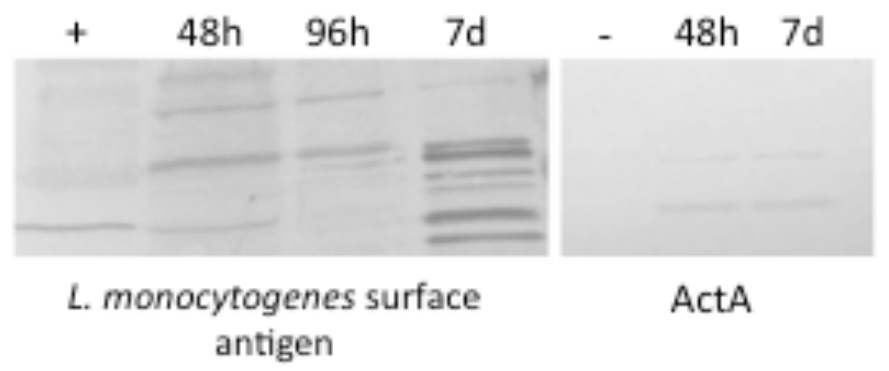

Figure 2 - Western blot detecting L. monocytogenes in zebrafish brain (surface antigen) and the detection of the ActA virulence factor (right). 